"kiss" — 2007/8/10 — 11:14 — page $99-\# 1$

\title{
The sum and difference of the areas of Napoleon triangles
}

\author{
SÁNDOR KISS
}

Abstract. The sum of the areas of the Napoleon triangles is the average of the areas of the three outward equilateral triangles on the sides of triangle $A B C$, and the differerence of these areas is the area of triangle $A B C$. In this paper we examine how to change these properties if we build on the sides of the triangle $A B C$, outwards and inwards, three similar triangles.

Key words and phrases: outer Napoleon triangle, inner Napoleon triangle.

ZDM Subject Classification: G05, G06.

\section{Introduction}

Let $L, M, N$ be the circumcenters of the equilateral triangles $B X C, C Y A$, $A Z B$ built outwards on the sides of an arbitrary triangle $A B C$ and $L^{\prime}, M^{\prime}, N^{\prime}$ the circumcenters of the equilateral triangles $B X^{\prime} C, C Y^{\prime} A, A Z^{\prime} B$ built inwards on the sides of the triangle $A B C$. In [7] we find the following properties:

"The sum of the areas of the Napoleon triangles $L M N$ and $L^{\prime} M^{\prime} N^{\prime}$ is the average of the areas of the three outward equilateral triangles on the sides of triangle $A B C$, and the differerence of these areas is the area of triangle $A B C$."

So

$$
\begin{aligned}
\sigma[L M N]+\sigma\left[L^{\prime} M^{\prime} N^{\prime}\right] & =\frac{\sigma[B X C]+\sigma[C Y A]+\sigma[A Z B]}{3}, \\
\sigma[L M N]-\sigma\left[L^{\prime} M^{\prime} N^{\prime}\right] & =\sigma[A B C]=\Delta,
\end{aligned}
$$


where $\sigma[L M N]$ denote the area of the triangle $L M N$.

In this paper we examine the changing of the relations (1) and (2) if we build on the sides of the triangle $A B C$, outwards and inwards, three similar triangles. In the next part we use the law of sines: if the sides of the triangle $A B C$ are $a$, $b$ and $c$ and the angles opposite those sides are $A, B$ and $C$, then

$$
\frac{a}{\sin A}=\frac{b}{\sin B}=\frac{c}{\sin C}=2 R,
$$

where $R$ is the radius of the triangle's circumcircle.

\section{The outward case}

We build on the sides of the triangle $A B C$ outwards the similar triangles with one another $B C D, C A E$ and $A B F$ in that way, that $B A F \varangle=\alpha=C A E \varangle$, $C B D \varangle=\beta=A B F \varangle, A C E \varangle=\gamma=B C D \varangle$ (Figure 1). Since $A+B+C=\pi=$ $\alpha+\beta+\gamma$, therefore $B D C \varangle=\alpha, C E A \varangle=\beta, A F B \varangle=\gamma$.

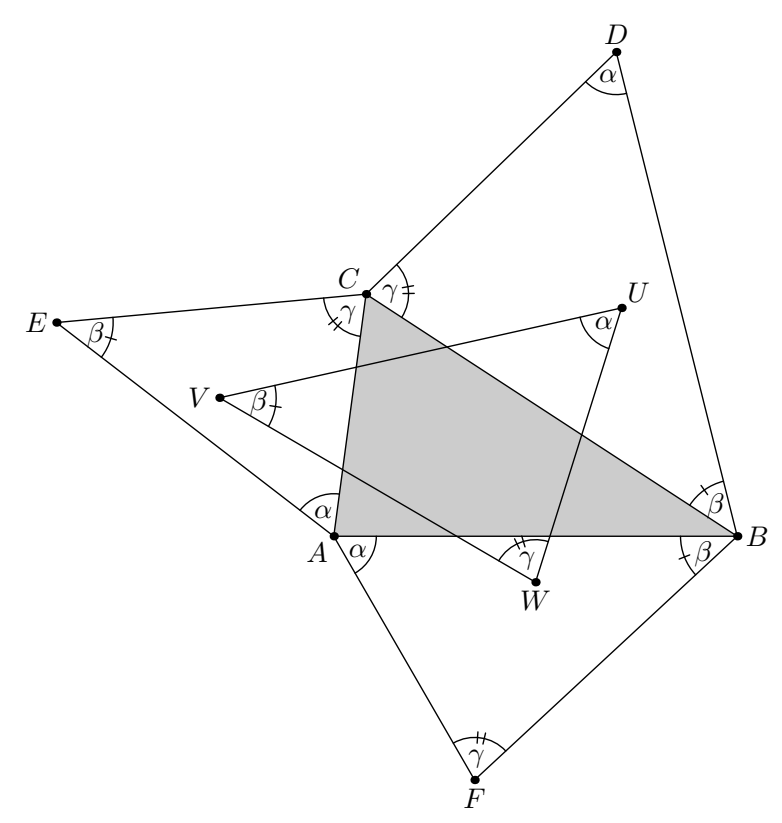

Figure 1 
Applying the law of sinus to the triangles $B C D, C A E$ and $A B F$, we obtain

$$
\begin{aligned}
& \frac{a}{\sin A}=\frac{C D}{\sin B}=\frac{B D}{\sin C}=2 R_{a}, \\
& \frac{C E}{\sin A}=\frac{b}{\sin B}=\frac{A E}{\sin C}=2 R_{b}, \\
& \frac{B F}{\sin A}=\frac{A F}{\sin B}=\frac{c}{\sin C}=2 R_{c},
\end{aligned}
$$

where $R_{a}, R_{b}$ resp. $R_{c}$ denote the circumradius of the triangle $B C D, C A E$ resp. $A B F$. The areas of the respective triangles are

$$
\begin{gathered}
\sigma[B C D]=\frac{B D \cdot C D \sin \alpha}{2}=\frac{a^{2} \sin \beta \sin \gamma}{2 \sin \alpha}, \\
\sigma[C A E]=\frac{A E \cdot C E \sin \beta}{2}=\frac{b^{2} \sin \gamma \sin \alpha}{2 \sin \beta}, \\
\sigma[A B F]=\frac{A F \cdot B F \sin \gamma}{2}=\frac{c^{2} \sin \alpha \sin \beta}{2 \sin \gamma} .
\end{gathered}
$$

Let $U, V$ resp. $W$ denote the circumcenter of the triangle $B C D, C A E$ resp. $A B F$ (Figure 1). The triangle $U V W$ is called the outer generalized Napoleon triangle. For computing the sides of the outer Napoleon triangle, we use the law of cosines.

Since $\frac{A C}{A V}=\frac{A F}{A W}=\frac{C F}{V W}=2 \sin \beta$ and $\frac{A E}{A V}=\frac{A B}{A W}=\frac{B E}{V W}=2 \sin \gamma$, then $\triangle A C F \sim \triangle A V W \sim \triangle A E B$, consequently $V A W \varangle=A+\alpha$. Similarly is justifiable then $\triangle B F C \sim \triangle B W U \sim \triangle B A D$ and $\triangle C D A \sim \triangle C U V \sim \triangle C B E$, therefore $W B U \varangle=B+\beta$ and $U C V \varangle=C+\gamma$.

We determine the side $V W$ of the triangle $U V W$ :

$$
\begin{aligned}
V W^{2} & =R_{b}^{2}+R_{c}^{2}-2 R_{b} R_{c} \cos (A+\alpha) \\
& =\frac{b^{2}}{4 \sin ^{2} \beta}+\frac{c^{2}}{4 \sin ^{2} \gamma}-2 \frac{b c}{4 \sin \beta \sin \gamma} \cos (A+\alpha) \\
& =\frac{4 R^{2} \sin ^{2} B}{4 \sin ^{2} \beta}+\frac{4 R^{2} \sin ^{2} C}{4 \sin ^{2} \gamma}-2 \frac{4 R^{2} \sin B \sin C}{4 \sin \beta \sin \gamma} \cos (A+\alpha) \\
& =\frac{R^{2}}{\sin ^{2} \beta \sin ^{2} \gamma}\left[\sin ^{2} B \sin ^{2} \gamma+\sin ^{2} C \sin ^{2} \beta-2 \sin B \sin C \sin \beta \sin \gamma \cos (A+\alpha)\right] .
\end{aligned}
$$

Let's introduce the following notation:

$$
\begin{aligned}
\lambda & =\lambda(\alpha, \beta, \gamma, A, B, C) \\
& =\sin \alpha \sin \beta \sin \gamma\left(\cot \alpha \sin ^{2} A+\cot \beta \sin ^{2} B+\cot \gamma \sin ^{2} C+2 \sin A \sin B \sin C\right) \\
& =\frac{\sin \alpha \sin \beta \sin \gamma}{4 R^{2}}\left(a^{2} \cot \alpha+b^{2} \cot \beta+c^{2} \cot \gamma+4 \Delta\right) .
\end{aligned}
$$


Are available the following conditional identities:

$$
\begin{aligned}
& \sin ^{2} B \sin ^{2} \gamma+\sin ^{2} C \sin ^{2} \beta-2 \sin B \sin C \sin \beta \sin \gamma \cos (A+\alpha)=\lambda, \\
& \sin ^{2} C \sin ^{2} \alpha+\sin ^{2} A \sin ^{2} \gamma-2 \sin C \sin A \sin \gamma \sin \alpha \cos (B+\beta)=\lambda, \\
& \sin ^{2} A \sin ^{2} \beta+\sin ^{2} B \sin ^{2} \alpha-2 \sin A \sin B \sin \alpha \sin \beta \cos (C+\gamma)=\lambda .
\end{aligned}
$$

We will proove the identity (9):

$$
\begin{aligned}
\sin ^{2} B & \sin ^{2} \gamma+\sin ^{2} C \sin ^{2} \beta-2 \sin B \sin C \sin \beta \sin \gamma \cos (A+\alpha) \\
= & \sin ^{2} B \sin (\alpha+\beta) \sin \gamma+\sin ^{2} C \sin (\alpha+\gamma) \sin \beta \\
& -2 \sin B \sin C \sin \beta \sin \gamma \cos (A+\alpha) \\
= & (\sin \alpha \cos \beta \sin \gamma+\cos \alpha \sin \beta \sin \gamma) \sin ^{2} B \\
& +(\sin \alpha \sin \beta \cos \gamma+\cos \alpha \sin \beta \sin \gamma) \sin ^{2} C \\
& -2 \sin B \sin C \sin \beta \sin \gamma \cos (A+\alpha) \\
= & \cos \alpha \sin \beta \sin \gamma\left(\sin ^{2} B+\sin ^{2} C\right)+\sin \alpha \cos \beta \sin \gamma \sin ^{2} B+\sin \alpha \sin \beta \cos \gamma \sin ^{2} C \\
& -2 \sin B \sin C \sin \beta \sin \gamma \cos (A+\alpha) \\
= & \cos \alpha \sin \beta \sin \gamma\left(\sin { }^{2} A+2 \cos A \sin B \sin C\right) \\
& +\sin \alpha \cos \beta \sin \gamma \sin ^{2} B+\sin \alpha \sin \beta \cos \gamma \sin ^{2} C \\
& -2 \sin B \sin C \sin \beta \sin \gamma(\cos A \cos \alpha-\sin A \sin \alpha) \\
= & \sin \alpha \sin \beta \sin \gamma\left(\cot \alpha \sin ^{2} A+\cot \beta \sin ^{2} B+\cot \gamma \sin ^{2} C+2 \sin A \sin B \sin C\right) \\
= & \lambda .
\end{aligned}
$$

Consequently $V W=\frac{R \sqrt{\lambda}}{\sin \beta \sin \gamma}$. Similarly we obtain the sides $W U$ and $U V$ :

$$
\begin{aligned}
V W & =\frac{1}{2} \sqrt{\frac{\sin \alpha}{\sin \beta \sin \gamma}\left(a^{2} \cot \alpha+b^{2} \cot \beta+c^{2} \cot \gamma+4 \Delta\right)}, \\
W U & =\frac{1}{2} \sqrt{\frac{\sin \beta}{\sin \gamma \sin \alpha}\left(a^{2} \cot \alpha+b^{2} \cot \beta+c^{2} \cot \gamma+4 \Delta\right)}, \\
U V & =\frac{1}{2} \sqrt{\frac{\sin \gamma}{\sin \alpha \sin \beta}\left(a^{2} \cot \alpha+b^{2} \cot \beta+c^{2} \cot \gamma+4 \Delta\right)} .
\end{aligned}
$$

Now we determine the angles of the triangle $U V W$ :

$$
\begin{aligned}
\cos V U W \varangle=\frac{U V^{2}+U W^{2}-V W^{2}}{2 U V \cdot U W} \\
=\left(\frac{R^{2} \lambda}{\sin ^{2} \alpha \sin ^{2} \beta}+\frac{R^{2} \lambda}{\sin ^{2} \gamma \sin ^{2} \alpha}-\frac{R^{2} \lambda}{\sin ^{2} \beta \sin ^{2} \gamma}\right) \frac{\sin ^{2} \alpha \sin \beta \sin \gamma}{2 R^{2} \lambda} \\
=\frac{\sin ^{2} \beta+\sin ^{2} \gamma-\sin ^{2} \alpha}{\sin ^{2} \alpha \sin ^{2} \beta \sin ^{2} \gamma} \cdot \frac{\sin ^{2} \alpha \sin \beta \sin \gamma}{2}
\end{aligned}
$$




$$
\text { "kiss" — 2007/8/10 — 11:14 — page 103 — \#5 }
$$

The sum and difference of the areas of Napoleon triangles

$$
\begin{aligned}
& =\frac{\sin ^{2} \beta+\sin ^{2} \gamma-\sin ^{2} \alpha}{2 \sin \beta \sin \gamma}=\frac{2 \cos \alpha \sin \beta \sin \gamma}{2 \sin \beta \sin \gamma} \\
& =\cos \alpha \Rightarrow V U W \varangle=\alpha .
\end{aligned}
$$

Similarly we obtain that $W V U \varangle=\beta$ and $U W V \varangle=\gamma$. So the triangle $U V W$ is similar to the external triangles $B C D, C A E$ and $A B F$.

The area of the triangle $U V W$ is

$$
\begin{aligned}
\sigma[U V W] & =\frac{U V \cdot U W \sin \alpha}{2}=\frac{R^{2} \lambda}{2 \sin \alpha \sin \beta \sin \gamma} \\
& =\frac{1}{8}\left(a^{2} \cot \alpha+b^{2} \cot \beta+c^{2} \cot \gamma\right)+\frac{\Delta}{2}
\end{aligned}
$$

Let $R_{O}$ be the circumradius of the triangle $U V W$ :

$R_{O}=\frac{U V \cdot V W \cdot W U}{4 \sigma[U V W]}=\frac{R^{3} \lambda \sqrt{\lambda}}{\sin ^{2} \alpha \sin ^{2} \beta \sin ^{2} \gamma} \cdot \frac{\sin \alpha \sin \beta \sin \gamma}{2 R^{2} \lambda}=\frac{R \sqrt{\lambda}}{2 \sin \alpha \sin \beta \sin \gamma}$.

Therefore

$$
R_{O}=\frac{1}{4} \sqrt{\frac{a^{2} \cot \alpha+b^{2} \cot \beta+c^{2} \cot \gamma+4 \Delta}{\sin \alpha \sin \beta \sin \gamma}}
$$

\section{The inward case}

We build on the sides of the triangle $A B C$ inwards the similar triangles with one another $B C D^{\prime}, C A E^{\prime}$ and $A B F^{\prime}$ in that way, that $B A F^{\prime} \varangle=\alpha=C A E^{\prime} \varangle$, $C B D^{\prime} \varangle=\beta=A B F^{\prime} \varangle, A C E^{\prime} \varangle=\gamma=B C D^{\prime} \varangle$ (Figure 2). Since $A+B+C=$ $\pi=\alpha+\beta+\gamma$, therefore $B D^{\prime} C \varangle=\alpha, C E^{\prime} A \varangle=\beta, A F^{\prime} B \varangle=\gamma$.

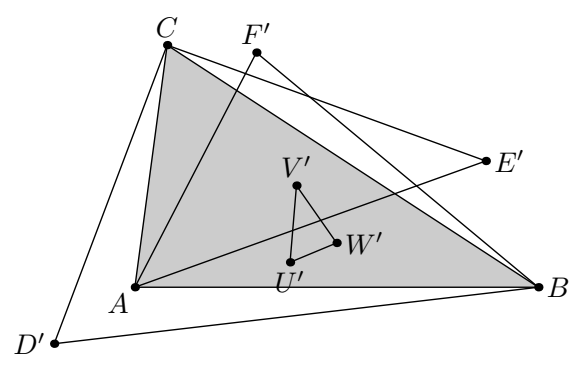

Figure 2 
Let $U^{\prime}, V^{\prime}$ resp. $W^{\prime}$ denote the circumcenter of the triangle $B C D^{\prime}, C A E^{\prime}$ resp. $A B F^{\prime}$ (Figure 2). The triangle $U^{\prime} V^{\prime} W^{\prime}$ is called the inner generalized Napoleon triangle. Here we introduce the following notation:

$$
\begin{aligned}
\lambda^{\prime} & =\lambda^{\prime}(\alpha, \beta, \gamma, A, B, C) \\
& =\sin \alpha \sin \beta \sin \gamma\left(\cot \alpha \sin ^{2} A+\cot \beta \sin ^{2} B+\cot \gamma \sin ^{2} C-2 \sin A \sin B \sin C\right) \\
& =\frac{\sin \alpha \sin \beta \sin \gamma}{4 R^{2}}\left(a^{2} \cot \alpha+b^{2} \cot \beta+c^{2} \cot \gamma-4 \Delta\right) .
\end{aligned}
$$

For determine the sides of the inner Napoleon triangle, here we use the following conditional identities:

$$
\begin{aligned}
& \sin ^{2} B \sin ^{2} \gamma+\sin ^{2} C \sin ^{2} \beta-2 \sin B \sin C \sin \beta \sin \gamma \cos (A-\alpha)=\lambda^{\prime}, \\
& \sin ^{2} C \sin ^{2} \alpha+\sin ^{2} A \sin ^{2} \gamma-2 \sin C \sin A \sin \gamma \sin \alpha \cos (B-\beta)=\lambda^{\prime}, \\
& \sin ^{2} A \sin ^{2} \beta+\sin ^{2} B \sin ^{2} \alpha-2 \sin A \sin B \sin \alpha \sin \beta \cos (C-\gamma)=\lambda^{\prime} .
\end{aligned}
$$

Since $V^{\prime} A W^{\prime} \varangle=|A-\alpha|, V^{\prime} B U^{\prime} \varangle=|B-\beta|$ and $U^{\prime} C V^{\prime} \varangle=|C-\gamma|$ therefore $V^{\prime} W^{\prime 2}=R_{b}^{2}+R_{c}^{2}-2 R_{b} R_{c} \cos (A-\alpha)$

$$
\begin{aligned}
& =\frac{b^{2}}{4 \sin ^{2} \beta}+\frac{c^{2}}{4 \sin ^{2} \gamma}-2 \frac{b c}{4 \sin \beta \sin \gamma} \cos (A-\alpha) \\
& =\frac{4 R^{2} \sin ^{2} B}{4 \sin ^{2} \beta}+\frac{4 R^{2} \sin ^{2} C}{4 \sin ^{2} \gamma}-2 \frac{4 R^{2} \sin B \sin C}{4 \sin \beta \sin \gamma} \cos (A-\alpha) \\
& =\frac{R^{2}}{\sin ^{2} \beta \sin ^{2} \gamma}\left[\sin ^{2} B \sin ^{2} \gamma+\sin ^{2} C \sin ^{2} \beta-2 \sin B \sin C \sin \beta \sin \gamma \cos (A-\alpha)\right] \\
& =\frac{R^{2} \lambda^{\prime}}{\sin ^{2} \beta \sin ^{2} \gamma} \Rightarrow V^{\prime} W^{\prime}=\frac{R \sqrt{\lambda^{\prime}}}{\sin \beta \sin \gamma} .
\end{aligned}
$$

Similarly we obtain the sides $W^{\prime} U^{\prime}$ and $U^{\prime} V^{\prime}$ :

$$
\begin{aligned}
V^{\prime} W^{\prime} & =\frac{1}{2} \sqrt{\frac{\sin \alpha}{\sin \beta \sin \gamma}\left(a^{2} \cot \alpha+b^{2} \cot \beta+c^{2} \cot \gamma-4 \Delta\right)}, \\
W^{\prime} U^{\prime} & =\frac{1}{2} \sqrt{\frac{\sin \beta}{\sin \gamma \sin \alpha}\left(a^{2} \cot \alpha+b^{2} \cot \beta+c^{2} \cot \gamma-4 \Delta\right)}, \\
U^{\prime} V^{\prime} & =\frac{1}{2} \sqrt{\frac{\sin \gamma}{\sin \alpha \sin \beta}\left(a^{2} \cot \alpha+b^{2} \cot \beta+c^{2} \cot \gamma-4 \Delta\right)} .
\end{aligned}
$$

The angles of the triangle $U^{\prime} V^{\prime} W^{\prime}$ are $V^{\prime} U^{\prime} W^{\prime} \varangle=\alpha, W^{\prime} V^{\prime} U^{\prime} \varangle=\beta$ and $U^{\prime} W^{\prime} V^{\prime} \varangle=\gamma$. So the triangle $U^{\prime} V^{\prime} W^{\prime}$ is similar to the internal triangles $B C D^{\prime}$, $C A E^{\prime}$ and $A B F^{\prime}$, too. 
The area of the triangle $U^{\prime} V^{\prime} W^{\prime}$ is

$$
\begin{aligned}
\sigma\left[U^{\prime} V^{\prime} W^{\prime}\right] & =\frac{U^{\prime} V^{\prime} \cdot U^{\prime} W^{\prime} \sin \alpha}{2}=\frac{R^{2} \lambda^{\prime}}{2 \sin \alpha \sin \beta \sin \gamma} \\
& =\frac{1}{8}\left(a^{2} \cot \alpha+b^{2} \cot \beta+c^{2} \cot \gamma\right)-\frac{\Delta}{2} .
\end{aligned}
$$

Let $R_{I}$ be the circumradius of the triangle $U^{\prime} V^{\prime} W^{\prime}$ :

$$
R_{I}=\frac{U^{\prime} V^{\prime} \cdot V^{\prime} W^{\prime} \cdot W^{\prime} U^{\prime}}{4 \sigma\left[U^{\prime} V^{\prime} W^{\prime}\right]}=\frac{R^{3} \lambda^{\prime} \sqrt{\lambda^{\prime}}}{\sin ^{2} \alpha \sin ^{2} \beta \sin ^{2} \gamma} \cdot \frac{\sin \alpha \sin \beta \sin \gamma}{2 R^{2} \lambda^{\prime}}=\frac{R \sqrt{\lambda^{\prime}}}{2 \sin \alpha \sin \beta \sin \gamma} .
$$

Therefore

$$
R_{I}=\frac{1}{4} \sqrt{\frac{a^{2} \cot \alpha+b^{2} \cot \beta+c^{2} \cot \gamma-4 \Delta}{\sin \alpha \sin \beta \sin \gamma}}
$$

4. The sum and difference of the areas of the generalized Napoleon triangles

Between the areas of the two generalized Napoleon triangles of the same triangle $A B C$ exist the following relations:

$$
\begin{aligned}
& \sigma[U V W]+\sigma\left[U^{\prime} V^{\prime} W^{\prime}\right]= \\
& \quad=\frac{1}{8}\left(a^{2} \cot \alpha+b^{2} \cot \beta+c^{2} \cot \gamma\right)+\frac{\Delta}{2}+\frac{1}{8}\left(a^{2} \cot \alpha+b^{2} \cot \beta+c^{2} \cot \gamma\right)-\frac{\Delta}{2} \\
& \quad=\frac{1}{4}\left(a^{2} \cot \alpha+b^{2} \cot \beta+c^{2} \cot \gamma\right), \\
& \sigma[U V W]-\sigma\left[U^{\prime} V^{\prime} W^{\prime}\right]= \\
& \quad=\frac{1}{8}\left(a^{2} \cot \alpha+b^{2} \cot \beta+c^{2} \cot \gamma\right)+\frac{\Delta}{2}-\frac{1}{8}\left(a^{2} \cot \alpha+b^{2} \cot \beta+c^{2} \cot \gamma\right)+\frac{\Delta}{2} \\
& \quad=\Delta .
\end{aligned}
$$

Summed up:

$$
\begin{aligned}
\sigma[U V W]+\sigma\left[U^{\prime} V^{\prime} W^{\prime}\right] & =\frac{1}{4}\left(a^{2} \cot \alpha+b^{2} \cot \beta+c^{2} \cot \gamma\right), \\
\sigma[U V W]-\sigma\left[U^{\prime} V^{\prime} W^{\prime}\right] & =\Delta=\sigma[A B C] .
\end{aligned}
$$

We can express the sum of the areas of the generalized Napoleon triangles with the areas of the external triangles $B C D, C A E$ and $A B F$ :

$$
\sigma[U V W]+\sigma\left[U^{\prime} V^{\prime} W^{\prime}\right]=\frac{1}{4}\left(a^{2} \cot \alpha+b^{2} \cot \beta+c^{2} \cot \gamma\right)
$$




$$
\begin{aligned}
=\frac{1}{2}( & \frac{\cos \alpha}{\sin \beta \sin \gamma} \cdot \frac{a^{2} \sin \beta \sin \gamma}{2 \sin \alpha} \\
& \left.\quad+\frac{\cos \beta}{\sin \gamma \sin \alpha} \cdot \frac{b^{2} \sin \gamma \sin \alpha}{2 \sin \beta}+\frac{\cos \gamma}{\sin \alpha \sin \beta} \cdot \frac{c^{2} \sin \alpha \sin \beta}{2 \sin \gamma}\right) \\
= & \frac{1}{2}\left(\frac{\cos \alpha}{\sin \beta \sin \gamma} \cdot \sigma[B C D]+\frac{\cos \beta}{\sin \gamma \sin \alpha} \cdot \sigma[C A E]+\frac{\cos \gamma}{\sin \alpha \sin \beta} \cdot \sigma[A B F]\right) .
\end{aligned}
$$

Consequently the relation (1) changes in the following way:

$$
\begin{aligned}
& \sigma[U V W]+\sigma\left[U^{\prime} V^{\prime} W^{\prime}\right]= \\
& \quad=\frac{1}{2}\left(\frac{\cos \alpha}{\sin \beta \sin \gamma} \cdot \sigma[B C D]+\frac{\cos \beta}{\sin \gamma \sin \alpha} \cdot \sigma[C A E]+\frac{\cos \gamma}{\sin \alpha \sin \beta} \cdot \sigma[A B F]\right) .
\end{aligned}
$$

The relation (2) remaines invariable.

\section{Special case}

If $\alpha=\beta=\gamma=\frac{\pi}{3}$ then the outer and the inner Napoleon triangles are equilateral and its areas are

$$
\begin{aligned}
\sigma[L M N] & =\frac{\sqrt{3}}{24}\left(a^{2}+b^{2}+c^{2}\right)+\frac{\Delta}{2}, \\
\sigma\left[L^{\prime} M^{\prime} N^{\prime}\right] & =\frac{\sqrt{3}}{24}\left(a^{2}+b^{2}+c^{2}\right)-\frac{\Delta}{2} .
\end{aligned}
$$

The circumradius of the outer resp. inner Napoleon triangle is

$$
\begin{aligned}
R_{O} & =\frac{\sqrt{a^{2}+b^{2}+c^{2}+4 \sqrt{3} \Delta}}{3 \sqrt{2}}, \\
R_{I} & =\frac{\sqrt{a^{2}+b^{2}+c^{2}-4 \sqrt{3} \Delta}}{3 \sqrt{2}} .
\end{aligned}
$$

Other arrangements of the triangles $B C D, C A E$ and $A B F$ are possible. We will present two of them (Figure 3 and Figure 4). To investigate the valabilities of the relations (27) and (2) in this cases is a possible subject for further researches. 


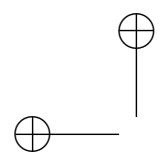

$$
\text { "kiss" — 2007/8/10 — 11:14 — page } 107 \text { — \#9 }
$$

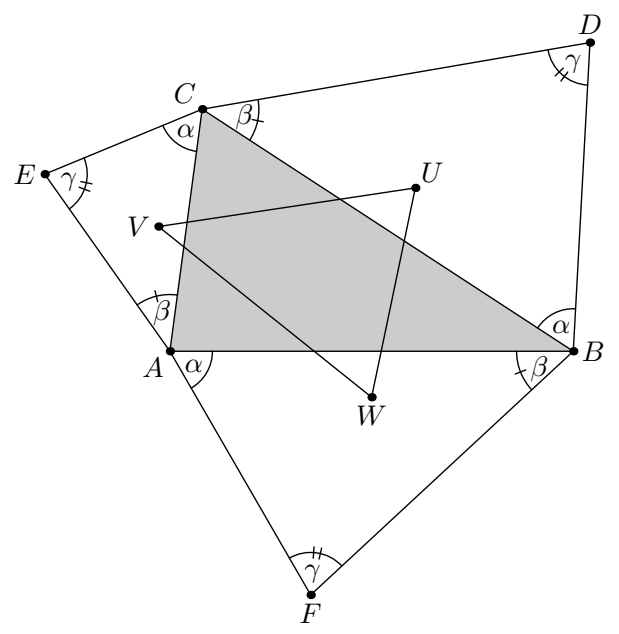

Figure 3

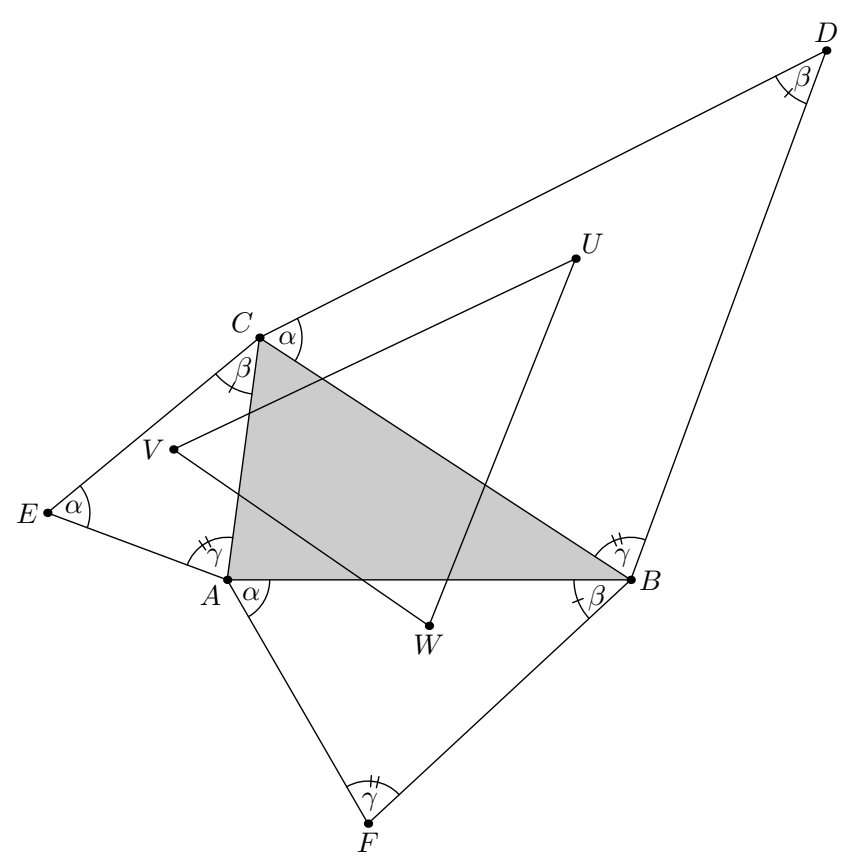

Figure 4 
"kiss" — 2007/8/10 — 11:14 — page 108 — \#10

\section{References}

[1] I. Belenkly, New Features of Napoleon's Triangles, J. Geom. 66 (1999), 17-26.

[2] H. S. M. Coxeter and S. L. Greitzer, Napoleon Triangles, §3.3, in: Geometry Revisited, Math. Assoc. Amer., Washington, DC, 1967, 60-65.

[3] J. F. Rigby, Napoleon Revisited, J. Geom 33 (1988), 129-146.

[4] I. M. Yaglom, Geometric Transformations I, Random House, New York, 1962, 38 and 93.

[5] E. W. Weisstein, Outer Napoleon Triangle, from MathWorld, http://mathworld. wolfram. com/OuterNapoleonTriangle.html.

[6] E. W. Weisstein, Inner Napoleon Triangle, from MathWorld, http://mathworld. wolfram. com/InnerNapoleonTriangle.html.

[7] J. E. Wetzel, Converses of Napoleon's Theorem, The Amer. Math. Monthly 99 (1992), 339-351.

SÁNDOR KISS

440095 SATU MARE

STR. AVRAM IANCU, NR. 58, SC. A, AP. 8

ROMANIA

E-mail: kissandor@clicknet.ro

(Received October, 2006) 